Meta

Journal des traducteurs

Translators' Journal

\title{
Le use de la common law : étude terminologique
}

\section{Gérard Snow}

Volume 47, numéro 2, juin 2002

Traduction et terminologie juridiques

URI : https://id.erudit.org/iderudit/008008ar

DOI : https://doi.org/10.7202/008008ar

Aller au sommaire du numéro

Éditeur(s)

Les Presses de l'Université de Montréal

ISSN

0026-0452 (imprimé)

1492-1421 (numérique)

Découvrir la revue

Citer cet article

Snow, G. (2002). Le use de la common law : étude terminologique. Meta, 47(2), 186-197. https://doi.org/10.7202/008008ar

\section{Résumé de l'article}

$\mathrm{Au}$ cours des vingt dernières années, une terminologie française normalisée de la common law s'est élaborée au Canada dans le cadre du Programme national de l'administration de la justice dans les deux langues officielles (PAJLO). À l'aide d'un dossier sur le terme use, l'auteur vise à donner un aperçu de la méthode employée au cours des travaux terminographiques préalables à la normalisation et des problèmes particuliers que pose la transposition linguistique en droit.
Ce document est protégé par la loi sur le droit d'auteur. L'utilisation des services d’Érudit (y compris la reproduction) est assujettie à sa politique d'utilisation que vous pouvez consulter en ligne.

https://apropos.erudit.org/fr/usagers/politique-dutilisation/ 


\title{
Le use de la common law: étude terminologique
}

\author{
GÉRARD SNOW \\ Université de Moncton, Moncton, Canada
}

\begin{abstract}
RÉSUMÉ
$\mathrm{Au}$ cours des vingt dernières années, une terminologie française normalisée de la common law s'est élaborée au Canada dans le cadre du Programme national de l'administration de la justice dans les deux langues officielles (PAJLO). À l'aide d'un dossier sur le terme use, l'auteur vise à donner un aperçu de la méthode employée au cours des travaux terminographiques préalables à la normalisation et des problèmes particuliers que pose la transposition linguistique en droit.
\end{abstract}

\begin{abstract}
Twenty years ago, work began on the standardization of a French-language terminology for the common law in Canada under the auspices of the National Program for the Integration of Both Official Languages in the Administration of Justice (POLAJ). Using his research on the term "use," the author provides an overview of the pre-standardization research and analysis process involved and the problems faced by terminologists in transposing legal concepts from one language to the other.
\end{abstract}

\section{MOTS-CLÉS/KEYWORDS}

terminologie, droit, juridique, common law, use

Le Programme national de l'administration de la justice dans les deux langues officielles (PAJLO) a été institué il y a vingt ans dans le but de coordonner et de soutenir les efforts consacrés à l'avancement de la common law en français (CLEF) et du droit civil en anglais au Canada. Administré par le ministère de la Justice du Canada et financé principalement par le ministère du Patrimoine canadien, il réunit une fois l'an des représentants de tous les organismes et institutions intéressés: facultés de droit, centres de jurilinguistique, associations de juristes, barreaux, organismes gouvernementaux. Il a (comme l'Académie française) son dictionnaire, qui recense le vocabulaire anglais de la common law, assorti de définitions et d'exemples d'utilisation, et recommande des équivalents français qui ont reçu l'aval de son comité de normalisation. La première édition de son Dictionnaire canadien de la common law (PAJLO 1997) recouvre les domaines du droit des biens et du droit successoral; une édition préliminaire contenait en plus la terminologie du droit de la preuve (PAJLO 1984).

Le comité de normalisation est formé en majorité de représentants des gouvernements régis par la common law qui publient leurs lois au complet dans les deux langues officielles (Canada, Nouveau-Brunswick, Ontario, Manitoba, Territoires du Nord-Ouest et Yukon), lesquels s'engagent à employer la terminologie française normalisée dans les documents qu'ils publient. Les dossiers sont préparés par un sous-comité technique composé d'experts en jurilinguistique ${ }^{1}$. La dissémination de 
la terminologie française normalisée de la common law s'opère naturellement par une multitude d'autres moyens: banques de terminologie, cours de droit, ouvrages didactiques et doctrinaux, plaidoiries, jugements rendus ou traduits en français, brochures d'information, polices d'assurance et contrats de toutes sortes, pour ne nommer que ceux-là.

L'objet du présent article est d'illustrer la démarche terminographique du PAJLO à l'aide d'un dossier que l'auteur a rédigé dans le cadre des travaux en droit des fiducies (trusts). Le dossier en question porte sur le terme use et ses composés. Comme d'habitude, l'étude se divise en deux grandes parties consacrées respectivement à l'analyse notionnelle (I) et à la recherche d'équivalents (II). Mais quelques mots d'abord sur le rôle qu'a joué le français dans le développement de la common law.

La common law en français n'est pas à proprement parler une invention récente, puisqu'en 1066, lorsque Guillaume le Conquérant accède au trône d'Angleterre, c'est le français parlé à l'époque en Normandie qui devient la langue du droit et de la justice du royaume. Ce ne sera qu'au XviII ${ }^{\mathrm{e}}$ siècle que l'anglais s'établira définitivement comme langue officielle de la justice, le français étant devenu, vers le $\mathrm{Xv}^{\mathrm{e}}$ siècle, le Law French ${ }^{2}$, en passant par l'anglo-normand. On n'a qu'à ouvrir un dictionnaire anglais de la common law pour constater le rôle prédominant qu'a joué l'ancien français dans le développement de sa terminologie: abandonment («abandonement»), abatement ("abatement»), abettor («abeteor»), abeyance ("abeance»)...

Il est bien évident qu'il ne suffit pas, pour « refranciser» la common law, d'exhumer les mots et expressions de l'ancien français. Il est vrai que le droit est une science, et que, comme toute discipline scientifique, elle comporte forcément un certain nombre de termes savants qui n'ont jamais eu d'autre sens qu'en droit («writ of fieri facias», "emphytéose») ou qui, courants autrefois, sont devenus obsolètes dans la langue courante («bote», « dol»). N’empêche que, contrairement aux autres disciplines scientifiques, le droit doit s'efforcer d'être à la portée de tous, puisque, comme dit l'adage, «nul n'est censé ignorer la loi». Aussi la vaste majorité des termes juridiques sont-ils des mots de tous les jours, qui prennent un sens plus ou moins technique en droit («title», «jugement»).

C'est ce même processus naturel que le terminologue cherchera à reproduire dans l'élaboration d'un vocabulaire français pour la common law. La langue française est riche de mots et tournures qui se sont avérés utiles dans les systèmes juridiques des multiples pays de la Francophonie. Or, ces éléments morphologiques du français juridique n'appartiennent pas exclusivement à la tradition civiliste; ce n'est pas parce qu'ils ont pris un sens précis dans le droit civil qu'il est interdit de leur en donner un autre en common law. C'est pourquoi à l'occasion le terminologue n'hésitera pas à recycler des termes juridiques français déjà en usage dans la tradition civiliste en les intégrant au réseau notionnel de la common law; ainsi, le terme «fiducie» est devenu, pour les juristes canadiens de la common law, l'équivalent français précis et exact du terme trust, quel que soit le sens particulier que «fiducie» puisse avoir dans la tradition romano-germanique. Mais la néologie de sens n'est évidemment qu'une des ressources à la disposition du terminologue; s'il le juge utile ou préférable, il recourra à la néologie de forme, soit en donnant nouvelle vie à un archaïsme («tenance» pour tenancy), soit - c'est le plus souvent le cas - en créant une nouvelle expression à partir de morphèmes français existants («fief simple résoluble» pour fee simple 
determinable). La seconde partie du dossier illustrera cette approche. Elle montrera aussi que la recherche terminologique ouvre le plus souvent la voie à plusieurs possibilités.

Concernant l'objet du dossier, il faut signaler dès l'abord que le mot use a des sens passablement différents en common law selon qu'il dérive du latin usus ou de l'anglo-normand oes, lui-même dérivé du latin opus. Dans sa première acception (ciaprès use 1), le terme relève du droit des biens et répond à la définition suivante (Jowitt 1977: 1836) :

In law, as in ordinary language, use denotes the act of employing a thing: thus, to cultivate land, to read a book, to inhabit a house, is to use those things.

L'antonyme de ce terme est non-use. Leurs équivalents normalisés respectifs sont «usage» et «non-usage». Une note du Dictionnaire canadien de la common law (PAJLO 1997: 639) précise :

Acception générale en droit des biens qui ne vise pas la notion de use en tant qu'ancêtre de la fiducie.

C’est précisément cette autre dimension du mot use qui fera l'objet du dossier qui suit.

\section{ANALYSE NOTIONNELLE}

Rappelons l'origine étymologique du terme use dans le contexte qui nous intéresse (Megarry et Wade 1975: 152):

The word use was derived not from the Latin usus but from the Latin opus in the phrase ad opus (on his behalf), via the Old French Aal oes or al uses and hence A to the use of it: thus land might be conveyed to and his heirs A to the use of B and his heirs, where nowadays we should say A in trust for B.

Dans ce nouveau contexte, nous croyons devoir distinguer trois nouvelles acceptions du mot use. Il y a d'abord le sens de use que l'on rencontre le plus souvent dans des tours comme to the use of (voir l'exemple précité). Nous l'appellerons use 2. Le mot use est aussi le nom donné à une institution particulière du droit anglais (ci-après use 3), laquelle est à l'origine d'une autre institution connue aujourd'hui sous le nom de fiducie ${ }^{3}$. Contrairement à celles qui précèdent, cette acception ainsi que celle qui suit n'ont d'application qu'en matière de biens réels ${ }^{4}$ (realty). Enfin, le mot use (ci-après use 4) désigne une forme particulière de use 2, à savoir l'intérêt foncier - reconnu en equity - qui échoit au bénéficiaire sous le régime du use 3. Employé dans ce dernier sens, le mot use, en anglais, s'emploie souvent au pluriel (ex. Statute of Uses, feofee to uses).

\section{A - Le use en tant que faculté de jouir d'une chose (use 2)}

On rencontre surtout cet usage, nous l'avons dit, dans des tours comme to the use of. Le Jowitt (1977: 1837) décrit ainsi cette acception :

Use also means benefit; thus, in an ordinary assignment of chattels the assignor transfers the property to the assignee for his absolute use and benefit. 
On pourrait être tenté à première vue de ramener cette acception à celle de use 1 , mais il existe une nuance sémantique importante, car, si l'acception de use 1 vise strictement l'emploi d'une chose, celle de use 2 implique le droit, l'intérêt ou la faculté d'en jouir. L'insistance des auteurs sur les différences étymologiques entre use $1^{\circ}$ et $2^{\circ}$ ne saurait être ignorée.

Cet usage est très fréquent dans la documentation, par exemple sous la forme suivante (Megarry et Wade 1975: 156):

to $\mathrm{A}$ and his heirs to the use of $\mathrm{B}$ and his heirs.

Comparons cet extrait à celui qui suit (Megarry et Wade 1975: 154) :

Until the testator died, the feofees held the land to his use; thereafter they held it to such uses as he had declared by his will.

Si la première occurrence du mot use vise exactement le même sens que celui visé dans l'extrait précédent, la seconde occurrence s'en distingue nettement, car le use ne vise plus la faculté d'une personne de jouir d'un bien, mais l'emploi qui sera fait de ce bien. Cet emploi pourrait être, par exemple, ce qu'on appelle un charitable use. Pourtant, dans l'extrait précité, le Jowitt (1977: 1837) poursuivait en disant:

In the expressions separate use, superstitious use, and charitable use, use has the same meaning [as in for his absolute use and benefit].

On peut douter de la justesse de cette observation. Le terme charitable use ramène à l'esprit le célèbre Charitable Uses Act d'Angleterre de 1601 et les autres lois qui s'en sont inspirées, tel le Mortmain and Charitable Uses Act de l'Ontario ${ }^{5}$, abrogé en 1982. Le texte ontarien contenait la disposition suivante:

1.C(2) The following shall be deemed to be charitable uses within the meaning of this Act,

(a) the relief of poverty;

(b) education;

(c) the advancement of religion; and

(d) any purpose beneficial to the community, not falling under the foregoing heads.

C'est ce qu'on appelle communément les catégories d'activités caritatives (heads of charity). Le use du charitable use, on le voit bien ici encore, ne désigne pas la faculté de jouir d'un bien, mais l'emploi qui en sera fait. Ce n'est donc pas le use $2^{\circ}$ dont il s'agit, mais le use $1^{\circ}$, c'est-à-dire celui qui a déjà reçu pour équivalent normalisé le mot « usage» (PAJLO 1997: 638).

\section{B - Le use en tant qu'institution (use 3)}

Le use dans ce sens-ci désigne le moyen qui a été élaboré au Moyen-Âge pour échapper à certains attributs de la tenure féodale en limitant le nombre d'occasions où il y avait transmission de saisine. Ainsi, lors d'un fieffement, au lieu de concéder un bienfonds à $\mathrm{A}$ à vie, puis à $\mathrm{B}$ et à ses héritiers - ce qui aurait entraîné une mutation de saisine d'abord au moment de la concession, puis au décès de A, puis de nouveau au décès de $B$ ainsi qu'à chaque concession faite par $B$ ou ses successeurs... et autant de perceptions de charges en faveur du suzerain —, il suffisait de le concéder en fief 
simple, disons, à une communauté religieuse to the use de $\mathrm{A}$ à vie, puis de $\mathrm{B}$ et de ses héritiers. Résultat: une seule transmission de saisine en faveur de la communauté religieuse (avec, en boni, un certain nombre d'allégements fiscaux), sans égard au nombre de fois que la jouissance réelle du bien-fonds pouvait changer de titulaire. Comme les tribunaux d'equity reconnaissaient l'intérêt véritable des bénéficiaires du use, par opposition au rôle purement nominal ou intermédiaire du concessionnaire en titre, les bénéficiaires n'étaient pas dépourvus de recours advenant un conflit avec le concessionnaire.

Le use, comme institution, a donné lieu à cette autre institution qu'on appelle la fiducie. C'est ainsi qu'on en est venu à désigner le use qui, par différents moyens plus ou moins détournés, échappait à l'application du Statute of Uses de 1535, cette fameuse loi d'Henri VIII dont le but était de transmettre la saisine au bénéficiaire (le cestui que use), faisant du coup disparaître l'intérêt du concessionnaire (le feoffee to uses). En principe, le Statute of Uses continue aujourd'hui de produire ses effets (sauf abrogation législative) dans les cas où le détenteur de la saisine pour autrui n'est pas à proprement parler un fiduciaire (trustee). La vente sur marché (bargain and sale), comme mode de transfert immobilier, a d'ailleurs pour fondement le use; or, la vente sur marché existe toujours, comme en fait foi le libellé classique de l'acte de transfert: "...by these presents does grant, bargain, sell, alien, release, convey and confirm, to have and to hold...». Par conséquent, plutôt que de dire que l'institution du use a cessé d'exister, il est plus juste de dire qu'elle ne subsiste plus que greffée ou incorporée à autre chose. En tout état de cause, on n'a pas tort de qualifier le terme use $3^{\circ}$ d'historique.

\section{C - Le use en tant qu'intérêt foncier reconnu en equity (use 4)}

Si on peut à juste titre qualifier d'historique le use comme institution, il serait sans doute téméraire d'en dire autant du use comme intérêt foncier, puisqu'on en parle encore dans nos lois ${ }^{6}$. Le use est devenu un intérêt foncier reconnu en equity à partir du XIV ${ }^{\mathrm{e}}$ siècle (Bastarache et Boudreau Ouellet 2001: 24).Voici ce qu'en dit le Jowitt (1977: 1837) :

The use or beneficial ownership was treated like an estate, and descended on the intestacy of the cestui que use to his heir in the same way as the land would have done. A use was also devisable by will, although the land was not.

Nous passerons maintenant en revue les principales expressions juridiques contenant le terme.

\section{Feoffment to uses}

Le transfert d'un domaine franc (freehold estate) par substitution (plutôt que par sous-inféodation) s'appelle un fieffement (feoffment). Le concédant s'appelle le fieffant (feoffor) et le concessionnaire, le fieffé (feoffee). Dans le cas du feoffment to uses - on dira plus tard conveyance to uses ou encore transfer to uses - le concessionnaire nominal s'appelle le feoffee to uses, tandis que le bénéficiaire en equity s'appelle le cestui que use $e^{7}$. L'appellation feoffor to uses, pour désigner le constituant d'un use, est plus rare. L'acte formaliste (deed) par lequel le use est constitué s'appelle deed 
to uses et l'opération, grant to uses, les parties étant respectivement le grantor to uses et le grantee to uses. Sous le régime d'enregistrement des titres de l'Ontario, on appelle owner to uses le concessionnaire dont le titre a été dûment enregistré à la suite d'un transfer to uses ${ }^{8}$.

\section{Execution of use}

Nous avons déjà parlé du Statute of Uses. Son effet, dit-on, est to execute the use. Voici comment Megarry et Wade (1975: 156) expliquent le processus :

The effect of (the Statute of Uses) was to execute ${ }^{\star}$ all uses to which it applied by taking the legal estate out of the feoffees to uses and converting the equitable interests of the cestui que use into corresponding legal estates. For example, if freehold land was conveyed to $A$ and his heirs to the use of $B$ and his heirs, the Statute executed the use, with the result that A was divested of all interest in the land, and B's equitable fee simple became legal.

*(Note en bas de page 3) Executed means effected or perfected, as opposed to executory (e.g., executory interests) which means not perfected. A conveyance executes an estate in the grantee; a contract to convey gives the intended grantee an interest which is executory until perfected by conveyance.

Le Statute of Uses a donc pour effet, aux yeux du législateur, d'améliorer le use, non pas en augmentant sa substance, mais en le transformant d'un domaine reconnu en equity en un domaine reconnu en common law, étant désormais assorti de la saisine. Naturellement, cette prétendue amélioration n'existe réellement qu'aux yeux du souverain, qui saura, lui, en profiter; car, du point de vue des parties en cause, la loi n'améliore en rien leur position, puisqu'elles se verront désormais assujetties aux charges féodales et fiscales qu'elles voulaient précisément éviter.

L'explication fournie dans la note en bas de page précitée porte à confusion dans la mesure où elle laisse entendre que le executed use serait le contraire du executory use. En réalité, le contraire du executed use est plutôt le unexecuted use, terme qui désigne tout use qui n'a pas subi l'effet du Statute of Uses. Nous reviendrons à la notion de executory use.

\section{Use upon use}

Le use upon use (et ses variantes use upon a use et use on a use) est un de ces stratagèmes évoqués plus haut qu'ont utilisés les avocats pour contourner le Statute of Uses. En voici un exemple: "To $A$ and heirs to the use of $B$ and heirs to the use of $C$ and heirs». Les tribunaux ont statué, dans pareil cas, qu'une fois appliquée au premier use, la loi avait épuisé ses effets, laissant intact («unexecuted») le second.

\section{Executory uses}

Création de l'equity, le use est, du coup, étranger aux catégories de la common law (au sens strict), si bien que tout use dont la jouissance n'est pas actuelle, mais différée c'est-à-dire tout future use —, ne pouvant pas être un résidu (remainder) — genre d'intérêt reconnu en common law seulement —, sera classé parmi les intérêts non 
réalisés (executory interests). Or, le grand avantage des intérêts non réalisés, comparativement aux résidus, c'est qu'ils échappent aux règles de la common law qui interdisent, entre autres, les springing interests et les shifting interests, ce qui fait que nous pouvons maintenant avoir des springing uses et des shifting uses, espèces de executory uses ${ }^{9}$.

Un springing interest est un intérêt éventuel (contingent interest) qui ne prend pas appui sur un domaine particulaire franc (particular estate of freehold), chose qu'interdit la common law comme suite logique à la règle interdisant la vacance de saisine. Si $\mathrm{X}$ tente d'aliéner son bien-fonds «à $\mathrm{A}$ pour dix ans, puis à $\mathrm{B}$ en fief simple, s'il est marié », il contrevient à cette règle, car il faudrait autrement que la saisine, qui ne peut être transmise qu'à B (A n'étant pas titulaire d'un domaine franc), demeure en suspens durant l'occupation temporaire de A et en attendant de voir si la condition suspensive (condition precedent) — savoir le mariage de B — va se réaliser. Pour que le résidu de B fût valide, il faudrait qu'il puisse jouir d'un springing interest, c'est-àdire d'un intérêt qui surgit à tout moment, ce que rejette péremptoirement la common law. Mais si le domaine de B est reconnu en equity plutôt qu'en common law (s'agissant, par exemple, d'un use), l'objection disparaît.

Un shifting interest, par ailleurs, est un intérêt futur qui, pour s'actualiser, doit interrompre le domaine particulaire qui le précède. La common law ne s'oppose pas au fait qu'un résidu éventuel puisse suivre un domaine particulaire anéantissable (defeasible), pourvu qu'il soit résoluble (determinable), c'est-à-dire qu'il prenne fin de façon naturelle, étant intrinsèquement périssable. Ce qu'elle n'admet pas, en revanche, c'est le fait qu'une condition résolutoire (condition subsequent) - donc extrinsèque - puisse avoir pour effet d'interrompre la durée naturelle du domaine particulaire en faveur d'un résiduaire (remainderman), créant ainsi un shifting interest, c'est-à-dire un intérêt qui bouge avant terme. Mais, de nouveau, si le domaine de B est reconnu en equity plutôt qu'en common law, il n'y aura pas de problème.

\section{LES ÉQUIVALENTS}

\section{A - Le use en tant que faculté de jouir d'une chose (use $\left.2^{\circ}\right)$}

Comme nous le faisions remarquer précédemment, on trouve le plus souvent le terme use 2 dans des tours comme to the use of ou to his/her use, qu'on rendrait tout naturellement en français par «au bénéfice de», "pour le compte de», "au profit de», «à l'usage de». Voici des passages illustrant cet emploi:

[...] le procédé [du use] consistait pour le tenant à transporter le bien pour son propre usage ou pour l'usage de ceux qu'il désignerait de son vivant ou par testament. (Bastarache et Boudreau Ouellet 2001:24)

En application [du Statute of Uses], si A concédait son domaine à C, au profit de B, la concession en faveur de B était réputée prendre effet immédiatement. (Grenon 1997: 12)

Il pourrait arriver que $u s e 2^{\circ}$ apparaisse dans un texte anglais en dehors des tours mentionnés, quoique nous n'en ayons pas constaté d'occurrences au cours de notre recherche, sinon dans des nomenclatures de dictionnaires. Le cas échéant, il nous paraîtrait logique d'appliquer la même solution, quant à son équivalent français, que s'il s'agissait du use $4^{\circ}$ (voir plus loin). 


\section{B - Le use en tant qu'institution (use $3^{\circ}$ )}

Traduire ou ne pas traduire: voilà la première question! On pourrait envisager d'abord de ne pas le traduire en français, suivant en cela l'usage prédominant (Bastarache et Boudreau Ouellet 2001 : 23; CTTJ 1982: 76; Grenon 1997: 12). Dans ce cas, le mot use, mot étranger, s'écrit normalement en italique et se prononce à l'anglaise [jus]. On ne fait pas habituellement l'élision (Bastarache et Boudreau 2001: 23; Grenon 1997: 12).

Une autre solution serait la création néologique du substantif « use » en français, sans italique et prononcé à la française $[\mathrm{yz}]$ — on écrirait donc «l'use» plutôt que «le use». Ce tour ne semble pas être passé dans l'usage.

Il est tout de même intéressant de noter qu'aucun terme de la common law n'a échappé jusqu'à présent, dans le processus de la normalisation, à la francisation ${ }^{10}$. Même «common law» et « equity», seuls cas où la graphie originale anglaise ait été conservée, sont considérés par le PAJLO comme francisés ${ }^{11}$, d'où l'absence de l'italique dans l'emploi des deux termes et l'élision devant « equity» (PAJLO 1997: 213). Ceci dit, le PAJLO n'a fait aucune recommandation concernant leur prononciation. Or, dans l'usage courant, on recourt à la prononciation anglaise, ce qui laisse supposer que la francisation soit imparfaite.

Entre les deux solutions qui précèdent, c'est la première, c'est-à-dire "le use », qui nous semble la plus acceptable. On peut douter en effet que le tour «l'use» (en romain et avec l'élision) passe jamais dans l'usage, peu importe qu'il soit prononcé à l'anglaise (comme « equity») ou à la française.

Reste encore une troisième solution: rendre use $3^{\circ}$ par un autre mot français. Dans ce cas, l'équivalent pourrait être le même que celui envisagé pour use $4^{\circ}$ (voir ci-après). Cependant, vu le caractère historique du terme use $3^{\circ}$, cette solution ne nous paraît pas nécessaire.

\section{C - Le use en tant qu'intérêt foncier reconnu en equity (use $4^{\circ}$ )}

Nous avons fait remarquer plus haut que le use en tant qu'intérêt foncier ne pouvait être considéré comme historique au même titre que l'institution (use $3^{\circ}$ ). Or, si le PAJLO (1997) a jugé bon de donner des équivalents français normalisés à des termes ésotériques comme fee tail ( fief taillé») et curtesy ("bénéfice du veuf»), comment ne pas le faire pour executed use ou conveyance to uses? En conséquence, bien qu'on puisse envisager use, c'est-à-dire l'absence de traduction, comme solution (Bastarache et Boudreau Ouellet 2001: 31; Grenon 1997: 11), nous serions plutôt portés, quant à nous, à chercher une traduction.

Comme pour use 2, "bénéfice» (Snow 2000: 49), "profit» et "usage ${ }^{12}$ se présentent à prime abord comme trois équivalents plausibles. À cette liste il faut ajouter «droit d'usage» (CTTJ 1980: 131) ${ }^{13}$, terme qui, dans les régimes civilistes, s'emploie de manière interchangeable avec le terme «usage» tout court ${ }^{14}$.

On peut commencer par écarter "profit», qui sert déjà à rendre le terme anglais profit en common law et serait source certaine d'équivoque. Le mot «bénéfice» est attrayant sur le plan sémantique, le use $4^{\circ}$ étant par essence un intérêt bénéficiaire. Par contre, «usage» et «droit d'usage» seraient sans doute plus évocateurs à la lumière de leur signification en droit civil. Voici la définition que donne le Grand Robert (1987: 9-604) du terme «usage» en droit français: 
III. Dr. Droit réel qui permet à son titulaire (v. Usager) de se servir d'une chose appartenant à autrui (v. aussi Usufruit). Cf. Code civil, art. 625-31. Avoir l'usage d'un bien. V. Jouir (dr.). Propriété* et usage. Usage des fruits d'un fonds: droit d'en prendre "autant qu'il (...) en faut pour ses besoins et ceux de la famille» (Code civil). C Usages forestiers: droit par lequel certaines personnes peuvent prendre certains produits dans une forêt. V. Affouage, glandage, pâturage. Usage d'une culture intercalaire (partage). Droit d'usage aux morts-bois, feuilles sèches... (soutrage). C Prêt $t^{*}$ à usage (v. Commodat).

Loin de nous l'idée de prétendre assimiler l'usage du droit civil au use. Nous donnons cette définition uniquement pour montrer que le mot "usage» a pris en régime civiliste un sens juridique qui s'éloigne du sens courant (emploi), et ce, malgré le fait que l'étymologie, dans ce cas-là, paraît être la même (à savoir usus), qu'il s'agisse du sens courant ou du sens juridique. Néanmoins, pour préserver la différence étymologique entre use-usus et use-oes, on pourrait être tenté d'écarter «usage» en faveur de "bénéfice». En tout cas, c'est principalement entre ces deux solutions que nous balançons. Voyons maintenant comment nous pourrions rendre les composés.

\section{Feoffment to uses}

Les traductions pertinentes que le PAJLO (1997) a déjà normalisées ont été mentionnées précédemment: «fieffement » (feoffment), «fieffant» (feoffor), «fieffé » (feoffee), «transport» (conveyance), «transfert» (transfer), «acte formaliste» (deed), "acte (formaliste) de transport» (deed of conveyance), «concession» (grant), «concédant» (grantor), «concessionnaire» (grantee), "propriétaire» et «titulaire» (owner). D'autres équivalents sont en voie de normalisation: "cestui que trust» et "bénéficiaire de fiducie» pour cestui que trust, "acte (formaliste) de fiducie» pour deed of trust.

Le problème qui reste consiste surtout à rendre le tour to uses. Si use 4 était rendu par «usage», il suffirait d'ajouter les mots «d'autrui» aux termes énumérés cidessus, ce qui donnerait par exemple «transport à usage d'autrui» (conveyance to uses). Cependant, lorsque le terme à traduire désigne le concessionnaire nominal (feoffee to uses, grantee to uses), on pourrait dire encore plus simplement «fieffé pour autrui » et «concessionnaire pour autrui », le mot « usage» étant sous-entendu. Si, en revanche, on retenait «bénéfice» pour use 4, le tour «transport à bénéfices» (Snow 2000 : 48) nous paraîtrait tout aussi acceptable que "transport à bénéfice d'autrui», sauf pour le cas du concessionnaire nominal, où il serait peut-être plus naturel de dire respectivement «fieffé pour autrui » (Snow 2000: 48) et «concessionnaire pour autrui ». Bastarache et Ouellet (2001: 26), quant à eux, ont employé «fieffé soumis au use».

Nous avons vu que le PAJLO prévoyait recommander deux solutions pour cestui que trust (dans le domaine des fiducies), savoir "cestui que trust» (en italique) et «bénéficiaire de fiducie». Si nous appliquons à l'espèce ce principe de la double solution, cestui que use pourrait soit rester sans traduction ("cestui que use»), soit se rendre par «usager», «bénéficiaire» (Snow 2000: 48) ou "bénéficiaire de use» (Bastarache et Ouellet 2001:25), selon la manière dont on a rendu use $4^{\circ}$. 


\section{Execution of use}

Le PAJLO (1997) a normalisé «intérêt non réalisé» pour executory interest. Les équivalents suivants sont en voie de normalisation dans le domaine plus général des fiducies: "exécution de la fiducie» et «exécution fiduciaire» pour execution of trust, " exécuter une fiducie» pour to execute a trust, "fiducie parfaite» pour executed trust et «fiducie imparfaite» pour les synonymes executory trust et imperfect trust.

Par rapport à l'opération du Statute of Uses, les termes clés sont execution of use, to execute the use, executed use et unexecuted use. Nous avons mentionné plus haut que l'effet du Statute of Uses était de bonifier le use en investissant le cestui que use de la saisine. Dans cette perspective, le verbe «parfaire» rendrait mieux la notion en cause que "réaliser», car il ne s'agit aucunement ici de la transformation d'un intérêt virtuel, tel un intérêt futur, en un intérêt actuel. De même, le executed use n'est pas un use qui serait passé de la virtualité à la réalité, mais un use qui a été "parfait» (au sens passif du verbe parfaire) par l'effet du Statute of Uses. À l'opposé, le unexecuted use est celui qui demeure «imparfait». Le terme execution se rendrait alors, dans ce contexte, par "perfectionnement».

Quant au terme executory use, il ne désigne pas, avons-nous fait remarquer, le use qui n'a pas été executed par l'effet du Statute of Uses, mais plutôt une espèce d'intérêt non réalisé (executory interest). Le qualificatif «non réalisé» nous semble donc approprié pour le rendre en français.

\section{Use upon use}

Le qualificatif «superposé» (Bastarache et Boudreau Ouellet 2001: 31) nous paraît intéressant, particulièrement lorsqu'il se trouve apposé au mot use ou à «droit d'usage ». En revanche, si "bénéfice » est retenu pour use $4^{\circ}$, "bénéfice sur bénéfice» nous semble acceptable. (Snow 2000:53). Nous écarterions le tour «du second degré»(CTTJ 1992).

\section{Executory uses}

Le springing use, avons-nous dit, est cette espèce de $u s e 4^{\circ}$ qui ne prend pas appui sur un domaine particulaire franc, si bien que, venu le moment de son actualisation, la saisine se trouve à surgir tout à coup, après avoir été tenue en suspens (in abeyance). Le qualificatif «suspendu» (Bastarache et Boudreau Ouellet 2001: 27; CTTJ 1982) n'est pas incorrect, car il s'agit effectivement d'un intérêt qui a subi l'effet d'une suspension, sauf qu'il manque quelque peu de spécificité, car ce n'est pas tant le fait que l'intérêt en question ait été suspendu (held in abeyance) qui en fait un springing use comme le fait qu'il reprenne vie. Le qualificatif «surgissant» (Snow 2000: 48) nous semble, à cet égard, plus fidèle par rapport à la métaphore exprimée en anglais.

Le shifting use, par contraste, est celui qui se déplace en interrompant prématurément le domaine particulaire qui le précède. Le problème avec le qualificatif «permutable» (Bastarache et Boudreau Ouellet 2001: 27; CTTJ 1982), c'est que la permutation signifie habituellement le changement réciproque de deux choses. Nous favorisons, quant à nous, l'adjectif «mouvant», quoique «mobile» serait aussi à considérer. 


\section{CONCLUSION}

Si le langage du droit en général évolue rapidement, cela est surtout vrai pour la common law en français, qui n'a pas encore atteint, pourrait-on dire, sa pleine autonomie. D'où l'importance de la poursuite des travaux terminologiques, qui nourrissent l'entreprise de normalisation. Nous espérons avoir réussi à donner un aperçu de sa méthode et des interrogations qu'ils soulèvent.

\section{NOTES}

1. Les membres du sous-comité actuel, dont les travaux portent sur le droit des fiducies, sont François Blais, Andrée Duchesne (coordonnatrice du PAJLO), Guy Jourdain, Réjean Patry et Gérard Snow.

2. Voici un exemple de Law French: "Tener in fee simple est a tener a ascú home ou feme, a luy et a ses heires et a ses assignes pur touts jours.» (Rastell 1579: 191)

3. Sur le droit des fiducies, voir Grenon 1997.

4. Sur le droit des biens réels, voir Bastarache et Boudreau Ouellet 2001; Snow 2000.

5. LRO 1980, chap. 297 - texte publié en version anglaise seulement.

6. Voir, par exemple, Nouveau-Brunswick, Loi sur les biens, L.R.N.-B. 1973, chap. P-19, art. 18: "Lorsque des biens-fonds sont restreints, par l'emploi de mots limitatifs dans un instrument, à certains uses, tous les uses prévus dans celui-ci, qu'ils soient exprès ou implicites en droit et qu'ils soient immédiats ou futurs, éventuels ou non réalisés, ou devant être déclarés en vertu d'un pouvoir que renferme cet instrument, prennent effet au moment et au fur et à mesure de leur naissance par l'effet du droit de tenure et de la saisine, et relativement au droit de tenure et à la saisine originairement dévolus à la personne qui est titulaire des uses, et toute saisine d'uses ou scintilla juris dont l'existence se poursuit à son égard ou à l'égard d'une autre personne n'est pas réputée nécessaire pour fonder des uses futurs, éventuels ou non réalisés ou leur donner effet de même qu'une telle saisine d'uses ou scintilla juris n'est pas réputée suspendue ni rester ou subsister à son égard ou à l'égard d'une autre personne.»

7. Autre exemple du Law French, forme abrégée, paraîtrait-il, de cestui a qui oes le feffment fut fait.

8. Ontario, Loi sur l'enregistrement des droits immobiliers, L.R.O. 1990, chap. L.5, par. 92(1).

9. À noter que dans certains textes américains, springing use est employé comme synonyme de executory use, et shifting use, de secondary use. Cet usage est étranger aux traditions anglaise et canadienne.

10. Voir cependant plus loin la traduction envisagée pour cestui que trust.

11. Le genre féminin a été également normalisé dans les deux cas.

12. Nouveau-Brunswick, Loi sur l'enregistrement foncier, L.N.-B. 1981, chap. L-1.1, art. 3.

13. Aussi Ontario, Loi sur les fiduciaires, L.R.O. 1990, chap. T.23: 22(2).

14. Code civil de France, art. 625 sq.; Code civil du Québec, art. 1172 sq.

\section{RÉFÉRENCES}

Bastarache, M. et A. Boudreau Ouellet (2001) : Précis du droit des biens réels, $2^{\mathrm{e} e ́ d ., ~ C o w a n s-~}$ ville $(\mathrm{Qc})$, Yvon Blais.

CTTJ (1980): Centre de traduction et de terminologie juridiques, Vocabulaire de la common law, tome I: Droit des biens - Procédure civile, Moncton, Université de Moncton, Faculté de droit.

CTTJ (1982): Centre de traduction et de terminologie juridiques, Vocabulaire de la common law, tome II : Droit des fiducies, Moncton, Université de Moncton, Faculté de droit.

Grand Robert (1987) : Le Grand Robert de la langue française, $2^{e}$ éd. par Alain ReY, Paris, Le Robert. Grenon, A. (1997): Les fiducies, coll. La common law en poche (vol. 5), Cowansville (Qc), Yvon Blais, et Bruxelles, Bruylant.

Jowitt (1977): Jowitt's Dictionary of English Law, $2^{\mathrm{e}}$ éd. par John Burke, Londres, Sweet \& Maxwell, 2 vol.

Megarry R. et H. W. R. Wade (1972): The Law of Real Property, 4 e éd., Londres, Stevens \& Sons Ltd. 
PAJLO (1984): Programme national de l'administration de la justice dans les deux langues officielles, Vocabulaire bilingue de la Common Law: Droit de la preuve (Terminologie française normalisée), Ottawa, Association du Barreau canadien.

PAJLO (1997): Programme national de l'administration de la justice dans les deux langues officielles, Dictionnaire canadien de la common law: Droit des biens et droit successoral (Terminologie française normalisée), Cowansville (Qc), Yvon Blais.

Rastell, J. (1579): An Exposition of Certaine Difficult and Obscure Wordes and Termes of The Lawes of This Realme, New York, Da Capo Press.

SNow, G. (2000): Les biens: Biens réels, coll. La common law en poche (vol. 11), Cowansville (Qc), Yvon Blais, et Bruxelles, Bruylant. 